\title{
Impact of Premature Ejaculation: The Psychological, Quality of Life, and Sexual Relationship Consequences
}

\author{
Raymond C. Rosen, PhD, ${ }^{*}$ and Stanley Althof, $\mathrm{PhD}^{\dagger \neq}$ \\ *New England Research Institutes, Watertown, MA, USA; ${ }^{\dagger}$ Center for Marital and Sexual Health of South Florida, West \\ Palm Beach, FL, USA; ${ }^{\ddagger}$ Case Western Reserve University School of Medicine, Cleveland, OH, USA \\ DOI: $10.1111 /$ j.1743-6109.2008.00825.x
}

\begin{abstract}
A B S T R A C T
Introduction. Premature ejaculation (PE) has been associated with a range of negative psychological effects, including anxiety, depression, and distress in men and their female partners.

Aim. To review evidence of the psychosocial concomitants of premature ejaculation in recent observational studies, and to consider the psychosocial and quality of life outcomes associated with PE, including effects on the partner relationship.

Main Outcome Measure. Psychosocial and quality of life consequences related to premature ejaculation.

Methods. A literature search was performed to retrieve publications relating to management or treatment of PE or male sexual dysfunction. Publications were included if they reported the impact of PE on the man, his partner or relationship, or the impact of male sexual dysfunction and included PE in the analysis.

Results. Eleven observational studies were selected. All these studies found evidence for an association between PE and adverse psychosocial and quality of life consequences, including detrimental effects on the partner relationship. Comparative analyses were restricted by major differences across the studies.

Conclusions. PE significantly negatively impacts men and their partners and may prevent single men forming new partner relationships. Men are reluctant to seek treatment from their physicians, although they may be more encouraged to do so through their partner's support and the availability of effective treatments. There is a need for validated diagnostic screening criteria and validated, reliable, brief patient-reported outcome measures that can be used to assess men with PE and their partners. These factors would allow further studies with more complete and accurate assessment of the impact of PE. Rosen RC, and Althof S. Impact of premature ejaculation: The psychological, quality of life and sexual relationship consequences. J Sex Med ${ }^{* *} ;{ }^{* *}:{ }^{* *}{ }^{* *}$.
\end{abstract}

Key Words. Ejaculatory Dysfunction; Interpersonal Difficulty; Sexual Dysfunction; Premature Ejaculation

\section{Introduction}

$\mathrm{P}$ remature ejaculation $(\mathrm{PE})$ has been associated with a range of negative psychological effects, including anxiety, depression and distress in men and their woman partners. [1-7]. The psychosocial or interpersonal distress that results from PE may affect men's quality of life (QoL) and partner relationships, their self-esteem and self-confidence, and can act as an obstacle to single men forming new partner relationships [1-4,8-14].

Currently, there are three key issues to be addressed in the study of PE.

\section{Definition and Diagnosis of PE}

The number of men presenting for treatment for $\mathrm{PE}$ is much lower than the prevalence of $\mathrm{PE}$, suggesting that it is an under-diagnosed condition, the prevalence estimates are too high, or men are reluctant to seek treatment $[2,4]$. These discrepancies in prevalence are likely to remain because, although there are several definitions of $\mathrm{PE}$, none are universally accepted, and validated diagnostic screening criteria have only just started to become available for clinical use [5-7,15-18]. Some recent proposals have been made for a statistical disease model for the formulation and diagnosis of $\mathrm{PE}$, 
although this approach remains controversial $[19,20]$.

The marked inconsistencies in the selection criteria and outcome measures across studies place constraints on the interpretation of results.

\section{Outcome Measures}

Intravaginal ejaculatory latency time (IELT) is an important variable in assessing the efficacy of treatments for PE [21]. As an outcome measure, IELT has the advantage of being relatively objective and reliable. However, measures of IELT alone would not fully capture the subjective distressing aspects of the disorder, and overlaps in IELT distributions have been found between PE and non-PE groups of men [5]. Distress and bother are important components of most current definitions of PE [17].

Two large observational studies in the United States and Europe have used validated patientreported outcome (PRO) measures to determine the level of distress and interpersonal difficulty in men with PE and their partners [5,7]. Further analysis of the U.S. study found that distress and control of ejaculation were the most important variables in determining the diagnosis of men with PE [22]. Additionally, this study showed that partner-reported distress was more indicative of $\mathrm{PE}$ in the man than either estimated or stopwatchmeasured IELT. Patrick et al. has shown that assessing the man's improvement in control of ejaculation and IELT together may be better predictors of the outcome of treatment than IELT alone [23].

In addition to documenting the magnitude of distress experienced by PE men and their partners, investigators have become increasingly interested in assessing the extent of $\mathrm{PE}$ impact in terms of psychological, interpersonal, quality of life, and sexual relationship consequences.

\section{Impact of PE Relative to Other Sexual Dysfunctions}

$\mathrm{PE}$ has been associated with an increased rate of-and association with-other comorbid male sexual dysfunctions $[2,10]$. Furthermore, it has always been thought that men with PE are less adversely affected than men with other sexual dysfunctions, such as erectile dysfunction (ED), but a recent study has disputed this assumption [6].

It is therefore important that the relationship of $\mathrm{PE}$ with other sexual dysfunctions and sexual satisfaction as well as a man's overall self-confidence and self-esteem be considered in assessing the psy- chosocial outcomes of the disorder. Comprehensive assessment and outcome measures should be used for this purpose.

In this evidence-based review, we focus on large, observational, and community-based studies that have assessed psychosocial aspects of PE. Where appropriate, we have added insights from some of the smaller studies. The data are presented as psychosocial and QoL concomitants of $\mathrm{PE}$, highlighting the impact on the partner and their relationship.

\section{Methods}

\section{Literature Search and Selection of Publications}

A comprehensive literature search was performed using the Medline, Embase (New York, NY, USA), Biosis (Philadelphia, PA, USA), Scisearch (Philadelphia, PA, USA), the Derwent Drug File (Philadelphia, PA, USA), and PsycINFO ${ }^{\circ}$ psychological databases (Washington, DC, USA) to identify all publications that included the following words in the title, abstract, or keywords: "management of premature ejaculation," "treatment of premature ejaculation," "male sexual dysfunction," "anxiety," "divorce," "depression," "interview," "quality of life," "scales," "questionnaires," "psychosocial," "social," and "relationships." The search was manually cross-referenced for all articles. Authors were contacted by e-mail for additional information.

All publications were reviewed and included if they reported the impact of $\mathrm{PE}$ on the man, his partner or relationship, or if they reported the impact of male sexual dysfunction and included PE in the analysis.

Eleven observational, non-interventional studies from 1997-2007 met the inclusion criteria and are summarized in Tables 1 and 2. The studies varied widely in design, population, and outcome measures. The majority of the studies included only men, though some included woman partners. Although the literature search identified several published clinical trials using various treatments for PE, these were not selected as most of them contained fewer subjects than the observational studies, and included only men with PE and their female partners.

\section{Recruitment of Subjects}

Most subjects were recruited using advertising in newspapers, radio, or clinics, followed by interview or completion of questionnaires [4-8,11]. Two 
studies surveyed large, pre-existing Internet panels $[1,2]$. Three studies used questionnaires in subjects registered with general practitioners, university alumni, or military school students $[3,9,11]$. One study was a retrospective review of patient records from earlier clinic consultations [10].

\section{Study Populations}

Two studies included both sexually functional subjects from the general or student population, and sexually dysfunctional subjects from outpatient clinics $[8,11]$. Four of the studies included only men $[1,2,4,11]$. Porst et al. was the only study to include both bisexual and homosexual men, in addition to heterosexual men [2].

Three studies included both male and female subjects $[3,8,10]$. Four studies included heterosexual men in stable relationships and their female partners [5-7,9].

The majority of the studies compared men with $\mathrm{PE}$ to men without PE $[1-3,5-9,11]$.

Men were studied from a number of countries, but only two studies included men in more than one country. Porst et al. studied men in three countries, and Giuliano et al. studied men and their partners in five European countries [2,7].

The number of men in each study ranged from 28 in a small qualitative study [4] to 12,133 in a large Internet survey [2].

The mean age of the men in the majority of the studies ranged from 35 to 46 years $[1,2,4-9,11]$. Three included men in their 60's and 70's [2-4].

\section{Definitions of PE}

Several different definitions of PE were used, but generally included concepts or themes specified by the DSM-IV criteria. These include the following: (i) persistent/recurrent ejaculation with minimum sexual stimulation before the person wishes (i.e., lack of control over ejaculation); (ii) ejaculation occurring before, on, or shortly after penetration (i.e., ejaculatory latency time shorter than desired); and (iii) consequences in the form of marked distress/interpersonal difficulty. Since all three elements of the DSM-IV are patient-reported and cannot be directly observed by clinicians, the differences in the patient selection criteria are mainly around how these criteria were operationalized for each study. Four studies included men's selfassessment, according to their own subjective criteria of distress without providing details $[3,4,8,9]$. Two studies used self-reported assessment criteria based on DSM-IV-TR $[1,16]$, and three studies used other diagnostic criteria $[2,7,10]$. Men in the remaining five studies were diagnosed by a clinician using DSM-IV-TR-related criteria $[5-7,10,11]$.

\section{Assessment of Impact of PE \\ Psychosocial Consequences}

Quantitative, validated patient- and partnerreported outcome measures were used in three of the seven studies that assessed the psychosocial consequences related to PE (Table 1) [5-7]. They included a question to specifically address the personal distress of men with $\mathrm{PE}$ and the impact on their female partners.

Two non-validated Internet surveys included assessment of related emotional responses as part of their evaluation of sexual functioning [1] or comorbid conditions [2].

The questionnaire used by Dunn et al. included the validated Hospital Anxiety and Depression (HAD) Scale [3]. Symonds et al. used thematic assessment of qualitative reviews, based on selfreporting of anxiety, embarrassment, and depression [4].

\section{Quality of Life Consequences}

Four studies measured the impact of $\mathrm{PE}$ on QoL (Table 1). McCabe evaluated the associations between intimacy, QoL, and sexual dysfunction [8]. Intimacy was measured using a validated Personal Assessment of Intimacy in Relationship Scale [PAIRS], with responses to 36 items in a 5-point Likert scale. QoL was measured using a validated Comprehensive Quality of Life (ComQoL) scale. Importance on each of the seven domains was assessed using a 5-point Likert scale. Satisfaction was assessed on each domain using a 7-point scale. A validated Sexual Dysfunction Scale (SEAR) was used to calculate a sexual dysfunction index using a weighted score based on severity, duration, and frequency.

Rowland et al. included a validated Self Esteem and Relationship (SEAR) questionnaire, a 14-item, self-administered questionnaire for men, to measure their confidence and self-esteem [6]. This study also included a validated Medical Outcomes Study SF-36, a 36-item, self-reported, generic health status questionnaire for men, in which scores were calculated for eight domains, reflecting overall health-related QoL.

Two studies used surrogate outcome measures to measure QoL $[3,4]$. Dunn et al. included a validated HAD scale. Symonds et al. used thematic analysis of qualitative interviews, including 


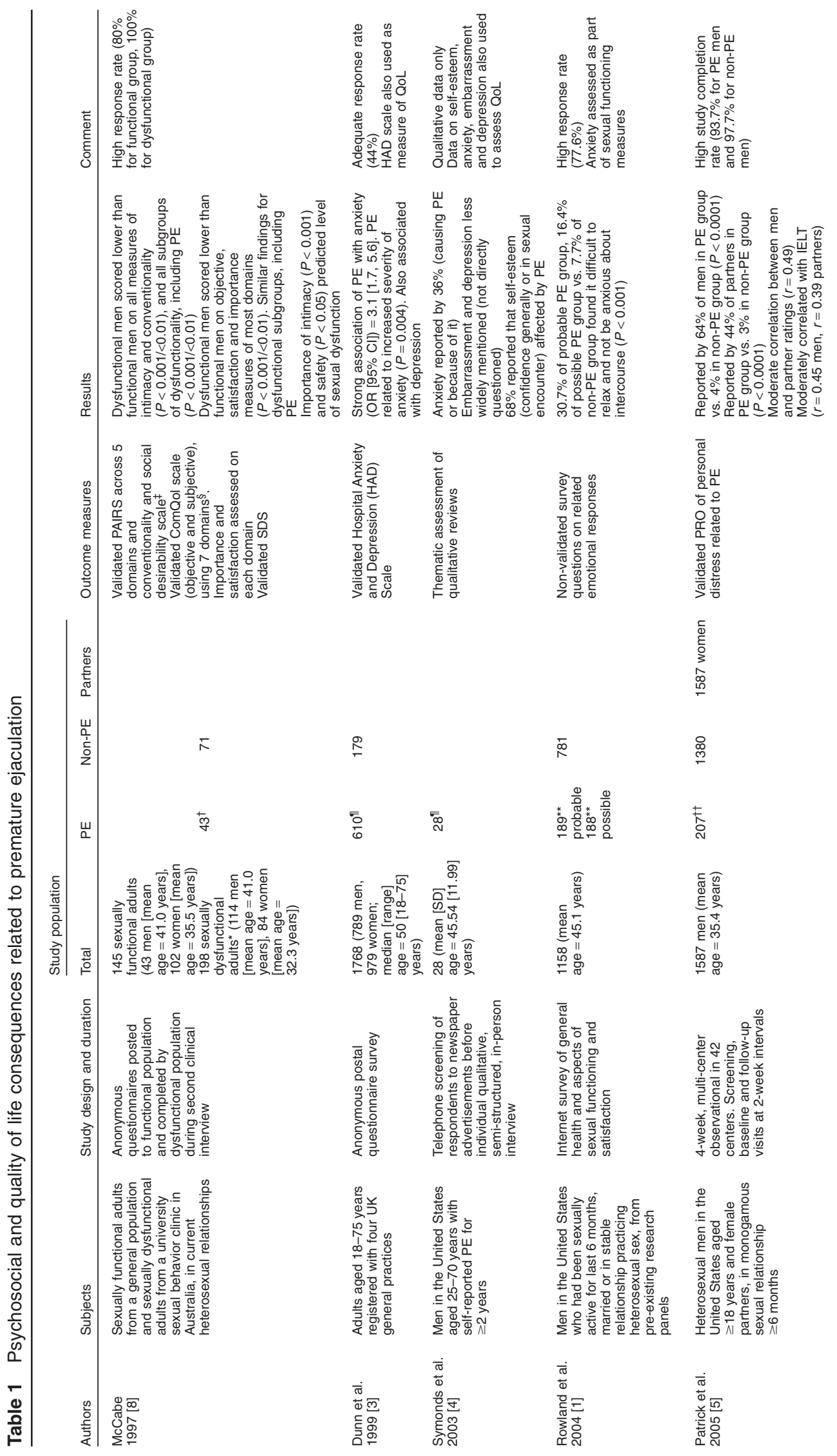



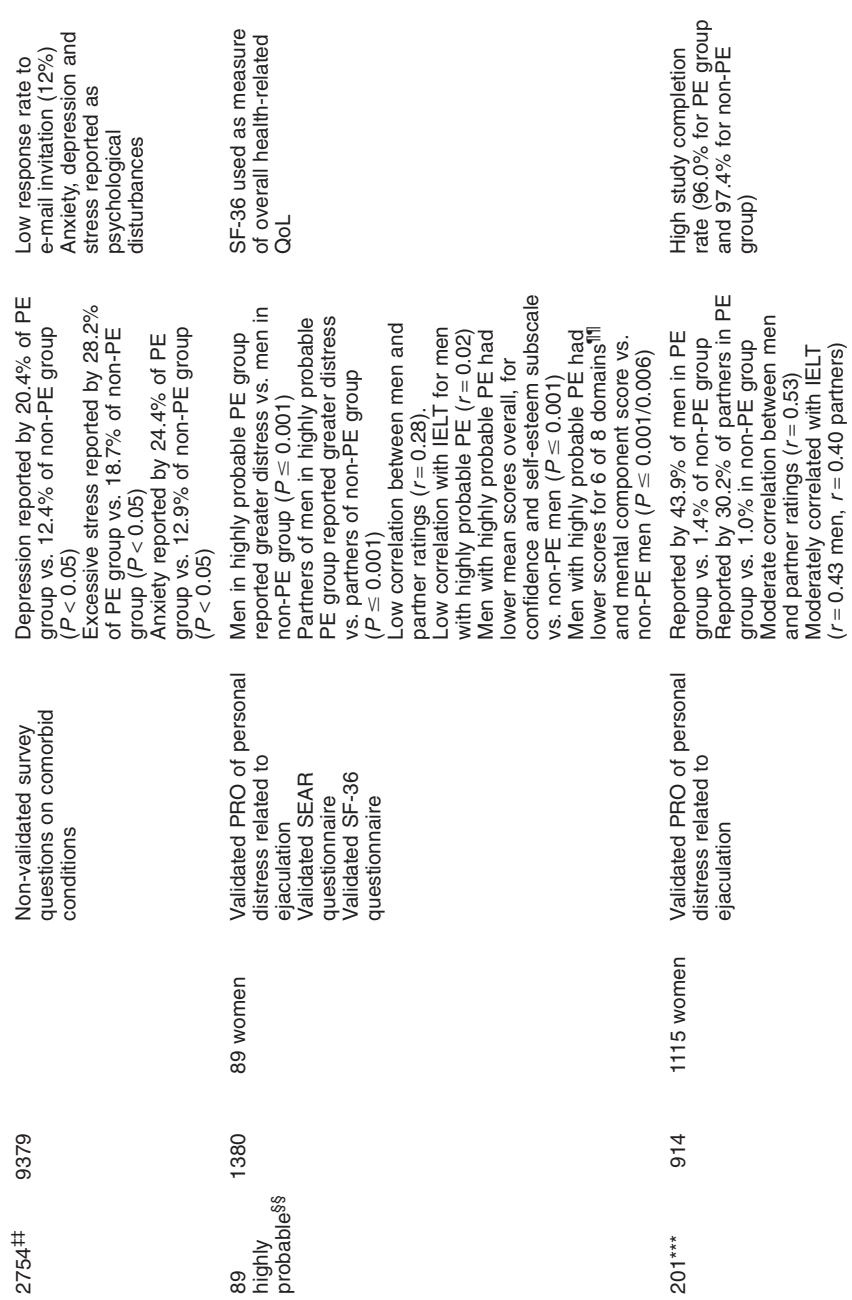

$\stackrel{5}{\circ}$

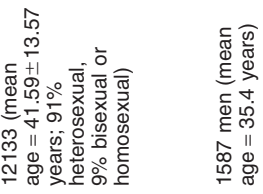

$\stackrel{*}{\stackrel{*}{*}}$

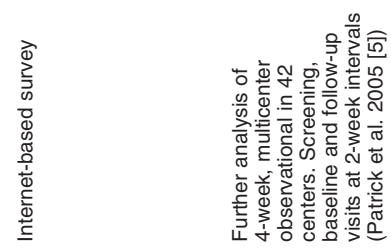

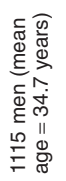
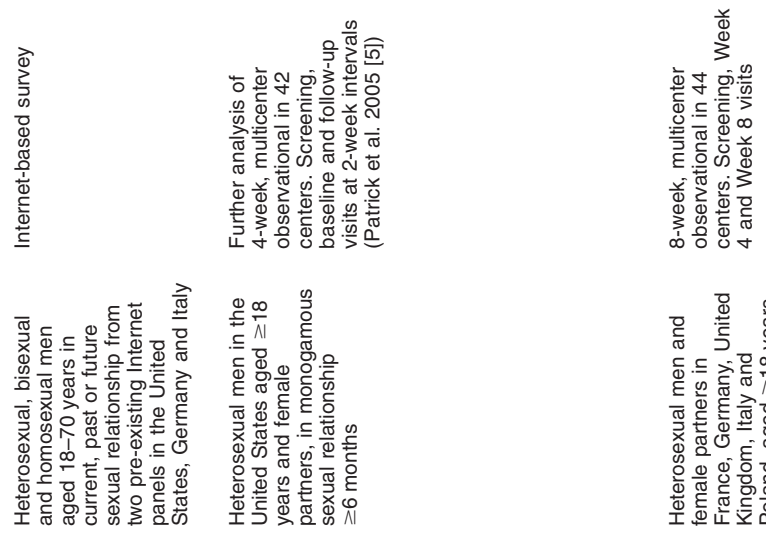

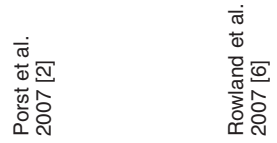
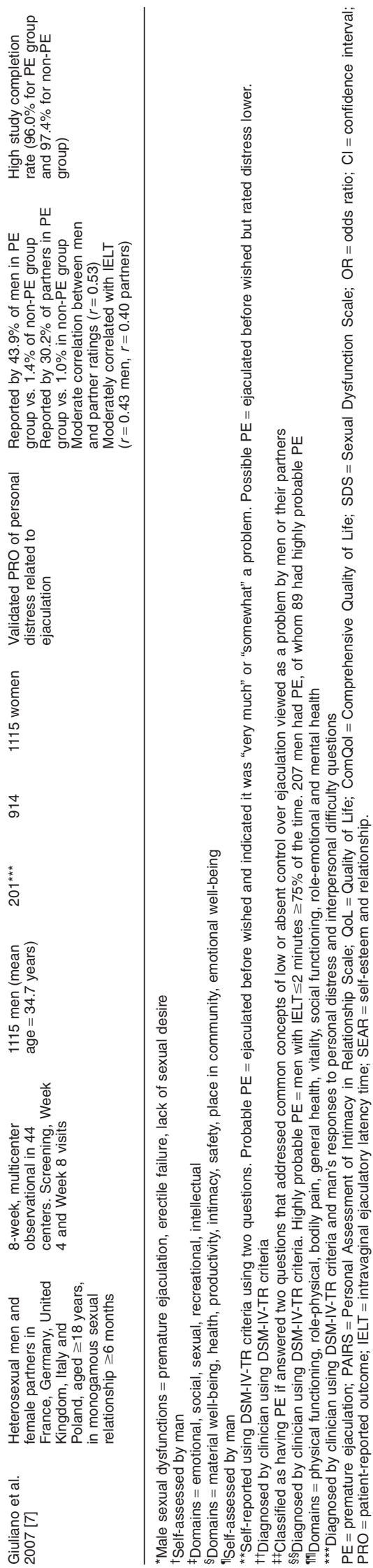
self-reporting of self-esteem, relationship issues, anxiety, embarrassment, and depression.

\section{Partner Relationship}

Of the eight studies that measured the impact of $\mathrm{PE}$ on the partner relationship, only four assessed both men and their partners (Table 2) [5-7,9]. The three more recent studies used validated quantitative measures of patient- and partner-outcomes to assess the interpersonal difficulty of men and their partners [5-7].

The smaller study by Byers et al. used a nonvalidated survey to assess perceptions of various $\mathrm{PE}$ criteria in men and their partners, and validated Global Measures of Relationship and Sexual Satisfaction Scales (GMREL and GMSEX) to assess their relationship and sexual satisfaction [9].

Three studies of men with PE included questions on partner-related issues $[1,4,11]$. Two included non-validated survey questions on sexual functioning and satisfaction related to their partner [1] or partner-related factors [11]. Symonds et al. used thematic assessment of qualitative reviews, based on self-reporting of relationship issues [4].

Riley et al. used non-validated assessment of diagnostic registers and case summaries from consultations for symptoms of sexually dysfunctional female patients who had male partners with PE [10].

\section{Results}

The psychosocial and QoL consequences related to $\mathrm{PE}$ and their impact on the partner relationship across the 11 studies, are summarized in Tables 1 and 2 .

\section{Psychosocial and Quality of Life Consequences Related to PE}

Table 1 shows that all seven studies that assessed psychosocial consequences revealed a high level of distress in men with PE [1-7], and in three of the studies [5-7], by their female partners.

In the large U.S. observational study by Patrick et al. using validated PRO measures, PE men reported substantially greater levels of distress than men without $\mathrm{PE}$ (2.69 vs. 0.69: 5-point scale ranging from $0=$ not at all distressed to 4 = extremely distressed), with extreme distress or "quite a bit" of distress being reported significantly more often by men with PE and their partners compared with the non-PE groups (64\% vs. $4 \%$ for men; $44 \%$ vs. $3 \%$ for partners, $P<0.0001$ ) [5].
Further analysis of these results showed that men with $\mathrm{PE}$ and their partners had premature ejaculation profile (PEP) scores for personal distress that were less than half those of the non-PE groups, i.e., worse $(P \leq 0.001)[6]$. Similarly, in a recent large European observational study, Giuliano et al. showed that substantially more men with $\mathrm{PE}$ and their partners reported that they were "extremely" or "quite a bit" distressed, compared with the non-PE groups (43.9\% vs. 1.4\% for men; $30.2 \%$ vs. $1.0 \%$ for partners) [7].

Both these large observational studies showed a moderate correlation between the couples' ratings for personal distress $(r=0.53$ [7], $r=0.49$ [5]) and IELT ( $r=0.45$ for men, $r=0.39$ for partners [5]; $r=0.43$ for men, $r=0.40$ for partners [7]).

In an earlier U.S. Internet survey, more men with $\mathrm{PE}$ reported that they had difficulty relaxing in sexual situations, and were more anxious about sexual intercourse compared with the non-PE group $(30.7 \%$ vs. $7.7 \%, P<0.001)$ [1]. Similarly, in a larger, more recent Internet survey in the United States, a significantly greater percentage of men with PE reported concomitant anxiety, depression, and psychological distress, compared with the non-PE group $(24.4 \%$ vs. $12.9 \%$ for anxiety, $P<0.05)$ [2].

A UK postal survey also showed a strong association of PE with anxiety (odds ratio 3.1) [3] and in their smaller U.S. qualitative study, Symonds et al. showed that over a third of men reported anxiety associated with $\mathrm{PE}$, with additional reports of embarrassment and depression [4].

Three studies looked at treatment-seeking behavior. Interestingly, two-thirds of the men studied by Symonds et al. had not consulted a physician because of embarrassment, nearly half believing that there was no treatment for PE, and therefore, would not seek medical help [4]. Almost $90 \%$ of the men in this study had tried treatment, which was mainly behavioral or psychological. In a study the same year, Byers et al. showed that only $4 \%$ of men with $\mathrm{PE}$ and $3 \%$ of their partners had ever sought help or information to resolve the problem, even if it was perceived by both partners [9]. Porst et al. was the only other study to assess treatment-seeking behavior by men [2]. Although over a fifth of the men with PE reported depression, stress or anxiety, only $9 \%$ had consulted a physician for their PE. Of these, over 90\% reported little or no improvement after treatment. Over half the men were aware that pharmacotherapy existed but only 13\% had used it. Strikingly, nearly two-thirds of the men with PE would 


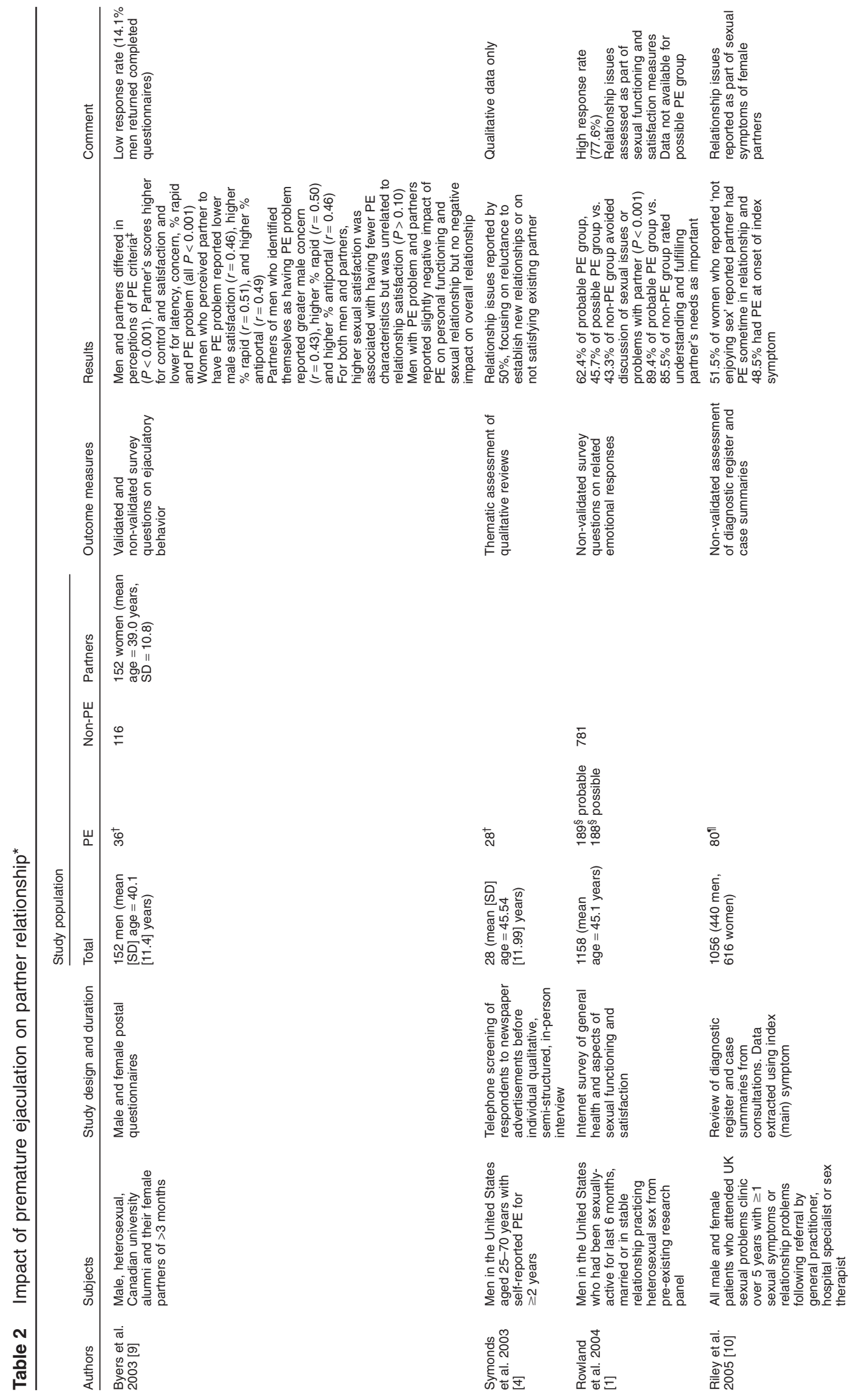




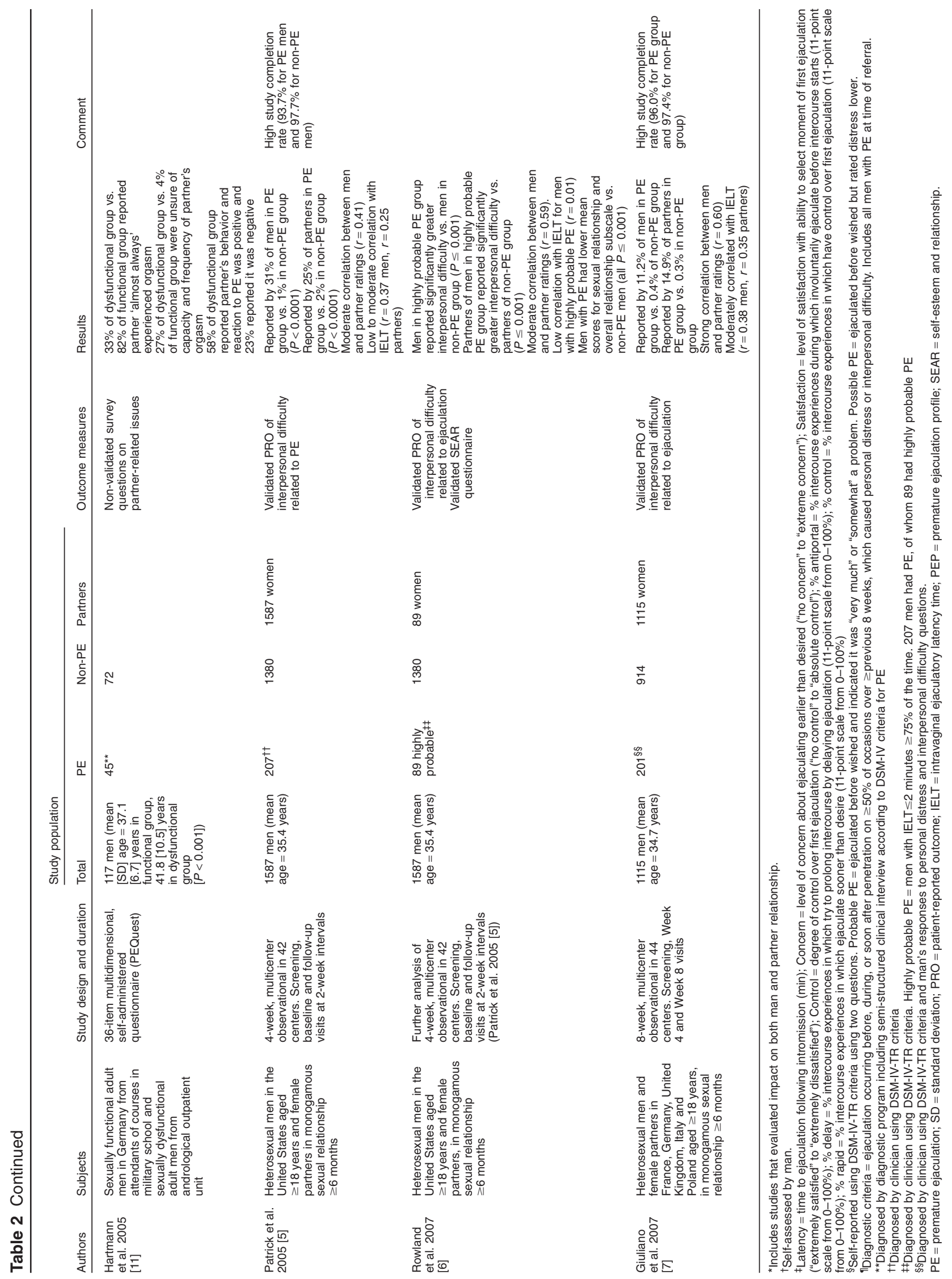

J Sex Med ${ }^{* *} ; * *: * *$ _** 
talk to a doctor about their condition if their partner suggested it, or if they knew of a pill to treat it. Nearly $15 \%$ had consulted a doctor because their partner had asked them to, and $75 \%$, because they wanted to better satisfy their partner sexually.

Four of the 11 studies showed that PE has a marked effect on the QoL of men (Table 1) $[3,4,6,8]$. McCabe showed that sexually dysfunctional men, including men with PE, scored lower on all aspects of intimacy (emotional, social, sexual, recreational, and intellectual) and had lower levels of satisfaction compared with sexually functional men $(P<0.001$ or $P<0.01)$ [8].

Rowland et al. showed that men with PE had significantly lower overall SEAR scores and lower confidence and self-esteem compared with non-PE groups (all $P \leq 0.001$ ) [6]. PE men rated their overall health-related QoL lower than men without $\mathrm{PE}(P \leq 0.001$ or $P \leq 0.006)$.

Symonds et al. and Dunn et al. showed a strong association between sexual confidence, anxiety, and in some cases, depression with PE $[3,4]$.

\section{Impact of PE on the Partner Relationship}

The eight studies in Table 2 show that PE greatly affected the men's relationship with their past, present, or future potential partners [1,4-7,9-11].

In their large observational study, Patrick et al. showed that a significantly greater proportion of men, and partners of men with PE, reported interpersonal difficulty compared with non-PE groups (31\% vs. $1 \%$ for men, $25 \%$ vs. $2 \%$ for partners, both $P<0.0001$ ) [5]. Further analysis of this study by Rowland et al. showed that men with PE and their partners reported PEP scores for interpersonal difficulty a third lower, i.e., worse, than those of the non-PE groups $(P \leq 0.001)[6]$. In the more recent European study, a consistent result was seen in more men with $\mathrm{PE}$ reporting interpersonal difficulty, compared with non-PE groups $(11.2 \%$ vs. $0.4 \%$ ) [7]. In this study, however, the partners' reporting of interpersonal difficulty was even greater $(14.9 \%$ vs. $0.3 \%)$.

The U.S. study showed moderate correlations between the ratings for interpersonal difficulty given by the men and their partners $(r=0.41)$ [5], while the European study showed a stronger correlation $(r=0.60)$ [7]. Interpersonal difficulty showed a weaker correlation with IELT in both studies ( $r=0.37$ for men, $r=0.25$ for partners [5]; $r=0.38$ for men, $r=0.35$ for partners [7]).

The importance of the impact of PE on the partner, further supports an earlier study by Byers et al. [9]. In their postal survey in Canada, women saw $\mathrm{PE}$ as less of a problem for their men partners than themselves.

Additionally, men with PE reported lower levels of sexual functioning and less confidence in their overall relationship, compared with non-PE controls $(P \leq 0.001)$ [6].

A U.S. Internet survey showed that a significantly greater percentage of men with $\mathrm{PE}$ reported that they avoided discussing sexual issues or problems with their partner, compared with the non-PE group $(62.4 \%$ vs. $43.3 \%, P<0.001)$ [1], even though most men with $\mathrm{PE}$ rated understanding and fulfilling their partner's needs as important.

Indeed in a small, non-comparative study in the United States, half the men with PE reported relationship issues, which generally focused on not satisfying their current partner or the reluctance of single men to establish new relationships [4].

A larger UK study that included the clinic records of female as well as male patients showed that about half of the female patients with sexual problems, such as "not enjoying sex" or anorgasmia, had partners with PE either at the onset of their problem or at some time in the relationship [10].

Hartmann et al. reported that $27 \%$ of dysfunctional men, including those with $\mathrm{PE}$, were unsure of the capacity and frequency of their partners' orgasm, compared with $4 \%$ of functional men [11]. The majority of these men reported that their partner's behavior and reaction to their dysfunction was positive.

Three studies assessed the impact of PE relative to other sexual dysfunctions, such as ED $[2,6,10]$. The large quantitative Internet survey by Porst et al. showed that men with $\mathrm{PE}$ were significantly more likely to self-report other sexual dysfunctions, such as orgasmic problems compared with men without PE (15.7\% vs. $4.4 \%)$, low libido (25.7\% vs. $9.1 \%)$, and $\mathrm{ED}(31.9 \%$ vs. $11.8 \%)$ (all $P<0.05)$ [2]. In a smaller study, $11 \%$ of men who had PE, reported that they had ED at the time that their PE had started [10].

In addition, a recent subanalysis of the large observational study by Patrick et al. suggested that men with PE are as psychologically distressed and affected as men with ED [6].

\section{Discussion}

We reviewed 11 observational studies that included over 19,000 men (4,470 of whom had PE) 
and 2,943 female partners. Previous reviews have looked at the psychological influence of $\mathrm{PE}$ on the man, the impact on his partner, and potential treatments for $\mathrm{PE}[13,14]$. In this review, we further analyze the psychosocial and QoL consequences of $\mathrm{PE}$ and its overall impact on partner relationships.

Overall, the studies confirmed a high level of personal distress reported by men with $\mathrm{PE}$ and their female partners [1-7], a considerable negative impact of $\mathrm{PE}$ on their QoL $[3,4,6,8]$, and partner relationships [1,4-7,9-11].

Comparative analyses of the data were restricted by major differences across the studies. These differences ranged from the methods used to recruit the subjects, population types, geographical location, sample size, age range, and definition of $\mathrm{PE}$, to the outcome measures used in the study. Only the minority of studies included heterosexual men in stable relationships and their female partners. Although this is consistent with the inclusion criteria of clinical trials, it creates problems when comparing these studies with the greater proportion of men-only studies. The impact of PE in single men may be greater and is certainly a barrier to them in establishing relationships [4]. This is an area that warrants further study.

Although different criteria were used to define $\mathrm{PE}$ across the studies, which resulted in a range of selection criteria, the negative impact of $\mathrm{PE}$ was consistent across studies. This is notable, given that the three studies that assessed treatmentseeking by men with $\mathrm{PE}$, all showed that most men were either reluctant or very reluctant to seek treatment $[2,4,9]$. Several reasons were given for this reluctance, including embarrassment and the belief that there were no treatments for PE. Significantly, one study showed that the partner was a key factor in the man's decision to seek treatment from a doctor for his PE [2]. That the impact of $\mathrm{PE}$ can be experienced by both partners in a sexual relationship, was an observation originally made by Masters and Johnson in 1970 [24]. The importance of the partner may provide the key to the problem of treatment-avoidance for $\mathrm{PE}$ and a direction for future research [2]. There is also a suggestion that when effective therapies become available, more men will seek treatment.

The minority of studies included quantitative, validated outcome measures $[3,5-8]$. It was, therefore, difficult to compare the results of these studies with the non-validated surveys, particularly those with surrogate outcome measures $[1,2,4,9-$ 11]. Other features of epidemiologic study designs that would improve the validity and generalizability of the findings would be to collect systematic information on comorbid conditions to allow for case-mix adjustment within study populations. External validity and generalizabilty is limited for those studies that did not clearly define how PE men were selected and/or employed invalidated scales for assessing the impact of the condition.

Several published clinical trials have used both IELT and PROs to measure the efficacy of various drug treatments on aspects of PE [25-28]. Although these studies show that treatment is effective in reducing the impact and distress of $\mathrm{PE}$ in men and their long-term partners, their subjects differ from "real world" populations, in that they are motivated to seek treatment and are selected according to the inclusion and exclusion criteria for the studies. However, there are no longitudinal data on the impact of PE to show the longterm effects of $\mathrm{PE}$ on their relationships and what happens to men with $\mathrm{PE}$ who do not receive treatment for their condition. In addition, unlike the clinical trial populations, none of the 11 articles applied a strict latency time (i.e., IELT) criteria for identifying PE patients. Although IELT may be useful for selecting more homogeneous clinical trial populations, its application to communitybased and/or epidemiologic studies for PE case identification may be limited by the assumption that time is uniformly linked to the degree of sexual stimulation across men [29].

This review has suggested that $\mathrm{PE}$ can have profound negative impact on the psychosocial consequences, QoL and interpersonal relationships of men with PE and their partners. Given the nature of consequences associated with $\mathrm{PE}$, it is notable that a review of the last 25 years of research revealed a decline in the proportion of psychological behavior articles with more frequent publication of biological and pharmacological articles [30]. Women are impacted by $\mathrm{PE}$ as much as their men partners-and possibly more. They were also an important factor in the men's decision to seek treatment for PE. It is, therefore, vital that physicians regard $\mathrm{PE}$ as a couple's problem and include the partner in its management whenever possible.

$\mathrm{PE}$ is a condition with a high level of psychological distress that may be as impactful as ED on the man and his partner. With a greater recognition of $\mathrm{PE}$ as a couple's problem, greater encouragement by the female partner and the availability of effective treatments, its impact on QoL and relationships should be significantly reduced. 


\section{Conclusions}

This evidence-based review of 11 studies shows that PE significantly impacts men and their partners negatively and can prevent single men from forming new partner relationships.

Although the studies reviewed demonstrate inconsistencies in terms of a standardized definition of $\mathrm{PE}$, validated diagnostic screening criteria, and validated PRO measures, they all provide a high degree of consistency in their conclusions. Clearly, PE has a substantial impact on both the man and his partner, and men are reluctant to seek treatment from their physicians. Much effort could, and perhaps should, be directed towards addressing these issues and encouraging further research in what happens to men with $\mathrm{PE}$ when their relationships are over.

\section{Acknowledgments}

The authors acknowledge the assistance of Brian Bulley, Carole Greenall, and Tara Symonds in preparation of this paper.

Corresponding Author: Raymond C. Rosen, $\mathrm{PhD}$, chief Scientist, New England Research Institutes, 9 Galen Street, Watertown, MA, USA 02472. E-mail: rrosen@neriscience.com

Conflict of Interest: Dr. Rosen is a consultant for Johnson and Johnson, Sanofi-Aventis, and Pfizer, Inc. Dr. Althof is a consultant for Johnson and Johnson, Sanofi-Aventis, and Pfizer, Inc.

\section{Statement of Authorship}

Category I

(a) Conception and Design

Raymond Rosen; Stanley Althof

(b) Acquisition of Data

Raymond Rosen; Stanley Althof

(c) Analysis and Interpretation of Data

Raymond Rosen; Stanley Althof

Category 2

(a) Drafting the Article Raymond Rosen; Stanley Althof

(b) Revising It for Intellectual Content Raymond Rosen; Stanley Althof

Category 3

(a) Final Approval of the Completed Article Raymond Rosen; Stanley Althof

\section{References}

1 Rowland DL, Perelman M, Althof SE, Barada J, McCullough A, Bull S, Jamieson C, Ho KF. Self- reported premature ejaculation and aspects of sexual functioning and satisfaction. J Sex Med 2004;1: 225-32.

2 Porst H, Montorsi F, Rosen RC, Gaynor L, Grupe $\mathrm{S}$, Alexander J. The premature ejaculation prevalence and attitudes (PEPA) survey: Prevalence, comorbidities, and professional help-seeking. Eur Urol 2007;51:816-23.

3 Dunn KM, Croft PR, Hackett GI. Association of sexual problems with social, psychological and physical problems in men and women: A crosssectional survey. J Epidemiol Community Health 1999;53:144-8.

4 Symonds T, Roblin D, Hart K, Althof SE. How does premature ejaculation impact a man's life? J Sex Marital Ther 2003;29:361-70.

5 Patrick DL, Althof SE, Pryor JL, Rosen RC, Rowland DL, Ho KF, McNulty P, Rothman M, Jamieson C. Premature ejaculation: An observational study of men and their partners. J Sex Med 2005;2:358-67.

6 Rowland DL, Patrick DL, Rothman M, Gagnon DD. The psychological burden of premature ejaculation. J Urol 2007;177:1065-70.

7 Giuliano F, Patrick DL, Porst H, La Pera G, Merchant S, Rothman M. Premature ejaculation: Results from a European observational study. Presented at: 21st Annual Congress of the European Association of Urology, Paris, France, April 5-8, 2006.

8 McCabe MP. Intimacy and quality of life among sexually dysfunctional men and women. J Sex Marital Ther 1997;23:276-90.

9 Byers ES, Grenier G. Premature or rapid ejaculation: Heterosexual couples' perceptions of men's ejaculatory behavior. Arch Sex Behav 2003;32:26170.

10 Riley A, Riley E. Premature ejaculation: Presentation and associations. An audit of patients attending a sexual problems clinic. Int J Clin Pract 2005; 59:1482-7.

11 Hartmann U, Schelowski M, Kruger TH. Cognitive and partner-related factors in rapid ejaculation: Differences between dysfunctional and functional men. World J Urol 2005;23:93-101.

12 Brock GB, Gajewski J, Carrier S, Bernard F, Lee J, Pommerville P. The prevalence and impact of premature ejaculation in Canada. Presented at: Annual Meeting of the American Urological Association, Anaheim, CA, May 19-24, 2007.

13 Althof SE. Prevalence, characteristics and implications of premature ejaculation/rapid ejaculation. J Urol 2006;175:842-8.

14 Althof SE. The psychology of premature ejaculation: therapies and consequences. J Sex Med 2006; 3:324-31.

15 World Health Organization. International statistical classification of diseases and related health problems. ICD-10. 1992;1:355-6. 
16 American Psychiatric Association. Diagnostic and statistical manual of mental disorders, 4th edition. text revision (DSM-IV-TR). Washington, DC: American Psychiatric Association; 2000

17 Montague DK, Jarow J, Broderick G, Dmochowski RR, Heaton JP, Lue TF, Nehra A, Sharlip ID. AUA guideline on the pharmacologic management of premature ejaculation. J Urol 2004;172:290-4.

18 Symonds T, Perelman MA, Althof SE, Giuliano F, Martin M, May K, Abraham L, Crossland A, Morris M. Development and validation of a premature ejaculation diagnostic tool. Eur Urol 2007;52:56573.

19 Waldinger MD, Schweitzer DH, Olivier B. On-demand SSRI treatment of premature ejaculation: pharmacodynamic limitations for relevant ejaculation delay and consequent solutions. J Sex Med 2005;2:121-31.

20 Waldinger MD, Schweitzer DH. Changing paradigms from a historical DSM-III and DSM-IV view toward an evidence-based definition of premature ejaculation. Part II-Proposals for DSM-V and ICD-11. J Sex Med 2006;3:693-705.

21 Rowland DL, Cooper SE, Schneider M. Defining premature ejaculation for experimental and clinical investigations. Arch Sex Behav 2001;30:235.

22 Rosen RC, McMahon CG, Niederberger C, Broderick GA, Jamieson C, Gagnon DD. Correlates to the clinical diagnosis of premature ejaculation: Results from a large observational study of men and their partners. J Urol 2007;177:1059-64.
23 Patrick DL, Rowland D, Rothman M. Interrelationships among measures of premature ejaculation: The central role of perceived control. J Sex Med 2007;4:780-8.

24 Masters W, Johnson V. Human sexual inadequacy. Boston, MA: Little Brown; 1970.

25 Kim SC, Seo KK. Efficacy and safety of fluoxetine, sertraline and clomipramine in patients with premature ejaculation: A double-blind, placebo-controlled study. J Urol 1998;159:425-7.

26 Atmaca M, Kuloglu M, Tezcan E, Semercioz A. The efficacy of citalopram in the treatment of premature ejaculation: a placebo-controlled study. Int J Impot Res 2002;14:502-5.

27 Safarinejad MR, Hosseini SY. Safety and efficacy of citalopram in the treatment of premature ejaculation: A double-blind placebo-controlled, fixed dose, randomized study. Int J Impot Res 2006;18:164-9.

28 Pryor JL, Althof SE, Steidle C, Rosen RC, Hellstrom WJG, Shabsigh R, Miloslavsky M, Kell S. Efficacy and tolerability of dapoxetine in the treatment of premature ejaculation: Integrated analysis of two randomized, double-blind, placebocontrolled trials. Lancet 2006;368:929-37.

29 Shabsigh R, Rowland D. The Diagnostic and Statistical Manual of Mental Disorders, 4th edition, text revision as an appropriate diagnostic for premature ejaculation. J Sex Med; 2007 Sep;4:1468-78.

30 Rowland D, Burek M. Trends in research on premature ejaculation over the past 25 years. J Sex Med 2007 Sep;4:1454-61. 